\title{
TABLETS FROM THE SIPPAR LIBRARY XI. THE BABYLONIAN ALMANAC
}

\author{
$B y$ KHALID SALIM ISMA'EL and A. R. GEORGE
}

The Babylonian Almanac is a text that gives abbreviated prescriptions for the days of the year. Since it was last edited, by René Labat in 1941 ("Un almanach babylonien", $R A 38,13-40$ ), new sources for the almanac have steadily accrued. Two more copies of the text have been identified among the tablets of the library discovered in the temple of Šmaš at Sippar by archaeologists of the University of Baghdad in 1986. Both are full almanacs, that is, they give an entry for all the days of the year, unfavourable as well as favourable. The two sources share a common format, with each month occupying one column of text, so that the first six months of the year occupy the obverse of the tablet and the last six months fill the reverse. They are, however, not exact duplicates, for on some days their entries differ.

A new edition of the almanac and other hemerological literature is in preparation by Alasdair Livingstone. Consequently the present publication is limited to making available the Sippar manuscripts without further discussion. ${ }^{1}$

\section{$\mathrm{SiA}$ \\ $\mathrm{SiB}$}

photograph: Figs. 1-2

cols. i-ix after a transliteration prepared in Baghdad in 1989; cols. $\mathrm{X}$-xii await study

\section{Month I: Nisan}

\begin{tabular}{|c|c|c|}
\hline 1 & $\mathrm{SiA}$ & 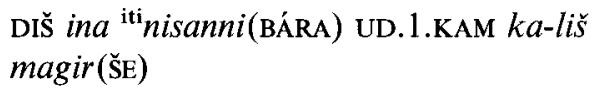 \\
\hline i 2 & $\mathrm{SiA}$ & ${ }^{12} \operatorname{din} a(\mathrm{DI})^{\top}$ là i-ger-rù \\
\hline i 3 & $\mathrm{SiA}$ & {$\left[3\right.$ nūna $\left.\left(\mathrm{KU}_{6}\right)\right]{ }^{\ulcorner} l a ̀$ ikkal $\left(\mathrm{GU}_{7}\right)^{1}$} \\
\hline i 4 & $\mathrm{SiA}$ & [4] $\left.{ }^{\lceil m i s ̌ i l(B A R}\right)^{\urcorner} \bar{u} m i(\mathrm{UD}) \operatorname{magir}(\mathrm{ŠE} . \mathrm{GA})$ \\
\hline i 5 & $\mathrm{SiA}$ & ${ }^{\prime} 5^{1}$ ümu(UD) là magru(ŠSE.GA) \\
\hline i 6 & $\mathrm{SiA}$ & ${ }^{1} 6^{1}$ mišil(BAR) $\bar{u} m i(\mathrm{UD}) \operatorname{magir}(\breve{\mathrm{SE}})$ \\
\hline i 7 & $\mathrm{SiA}$ & ${ }^{7} 7^{1} t e-s u-u$ \\
\hline i 8 & $\mathrm{SiA}$ & '81 sà-kap lúnakri(KÚR) \\
\hline i 9 & $\mathrm{SiA}$ & 9 ana sūqi(SILA) là uṣ̦i(È) șaltu(LÚ.NE) \\
\hline 10 & $\mathrm{SiA}$ & 10 șabāt eqli(A.Š̀̀.DAB) \\
\hline 11 & $\mathrm{SiA}$ & $11 h u-u d l i b-b i$ \\
\hline 12 & $\mathrm{SiA}$ & $12 \check{s}^{\prime} \bar{e}^{\prime} a\left(\mathrm{~S}_{\mathrm{E}}\right)$ là inaddin(SUM) șitu(ZI.GA) \\
\hline 13 & $\mathrm{SiA}$ & $\begin{array}{l}13 \text { aššata (DAM) là irašši(TUK) } \\
\left.{ }^{r} \text { balta (TÉš) }\right)^{-}-[s ̌ u(\mathrm{BI}) \text { itabbal(TÙM)] }\end{array}$ \\
\hline i 14 & $\begin{array}{l}\mathrm{SiA} \\
\mathrm{SiB}\end{array}$ & $\begin{array}{l}14 \text { ina di-[ni }]^{\top} \operatorname{magir}(\text { ŠE.GA })^{\top} \\
{[\ldots] \mathrm{x}}\end{array}$ \\
\hline i 15 & $\begin{array}{l}\mathrm{SiA} \\
\mathrm{SiB}\end{array}$ & $\begin{array}{l}15 \text { ma-[mit }] \text { isabbat (DAB)-su } \\
{[\ldots]-s i \text { ? }}\end{array}$ \\
\hline i 16 & $\begin{array}{l}\mathrm{SiA} \\
\mathrm{SiB}\end{array}$ & $\begin{array}{l}16 \text { ernetta }([\text { Ù].MA) kušud(SÁ.sá) } \\
{[\ldots] \text { TU? x }}\end{array}$ \\
\hline 17 & $\mathrm{SiA}$ & 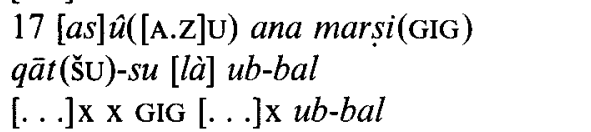 \\
\hline
\end{tabular}

II In Nisannu, day one: completely favourable 2: one must not go to law [3:] one must not eat [fish] [4:] midday is favourable 5: unfavourable day

6: midday is favourable

7: confusion

8: repulsion of the enemy

9: one should not go out in to the street: affray

10: seizure of a field

11: happiness

12: one must not barter grain: losses

13: one must not acquire a wife: [she will take away one's] dignity 14: it is favourable in a lawsuit

15: a curse might seize one

16: achieve an aim

17: doctor must not treat patient

\footnotetext{
${ }^{1}$ Both tablets are published here by permission of the Directorate-General of Antiquities and the University of Baghdad. We wish to record our thanks to Farouk Al-Rawi
}

and A. Livingstone for help with the preparation of this article. 


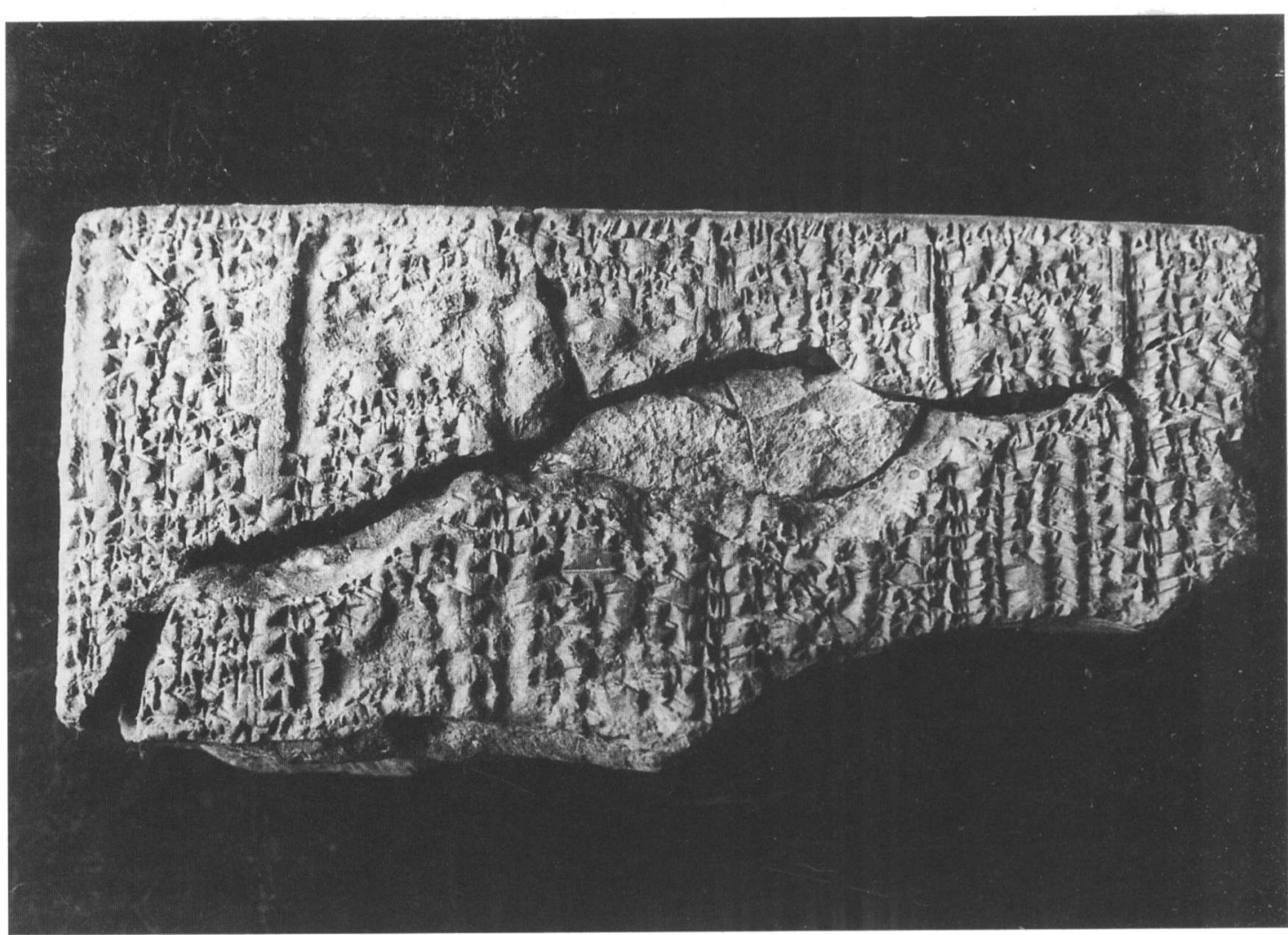

Fig. 1 IM 132596 obv.

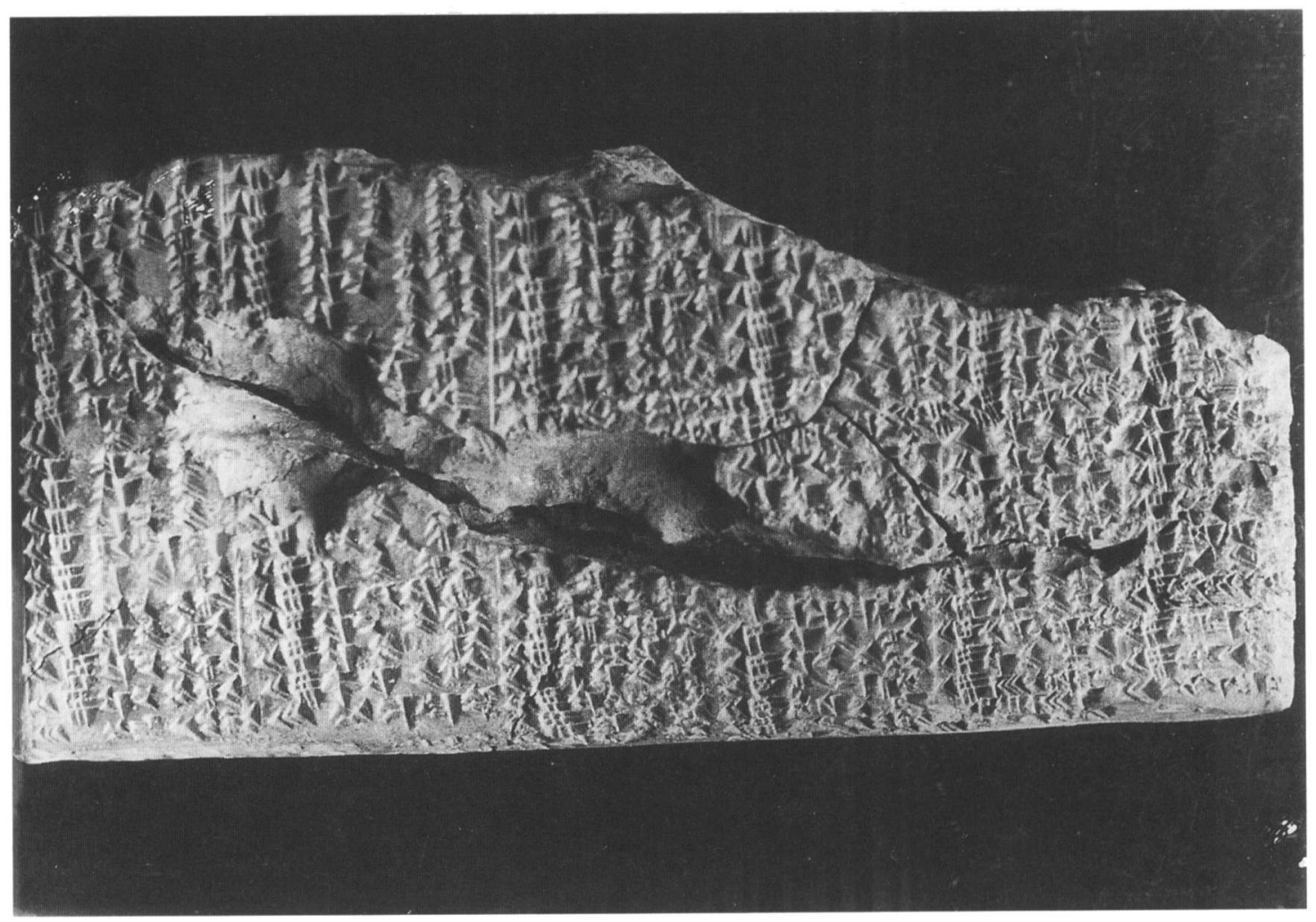

Fig. 2 IM 132596 rev. 


\begin{tabular}{|c|c|c|}
\hline i 18 & $\mathrm{SiA}$ & {$[18 \times] \times \times \times x$} \\
\hline & $\mathrm{SiB}$ & {$[18$ hi-bil-tu]m ussi(È) } \\
\hline i 19 & $\mathrm{SiB}$ & [19 bi-ki]-tum \\
\hline i 20 & $\mathrm{SiB}$ & {$[20 \ldots]$ HAR? DAB } \\
\hline i 21 & $\mathrm{SiB}$ & {$[21 \ldots]-i n ?-n i$} \\
\hline i 22 & $\mathrm{SiB}$ & {$[22 \ldots]$ ŠE.GA } \\
\hline i 23 & $\mathrm{SiB}$ & {$[23 \ldots]$ ŠE.GA } \\
\hline i 24 & $\mathrm{SiB}$ & {$[24 \ldots]$ HAR? } \\
\hline i $25-30$ & & not extant on eithe \\
\hline
\end{tabular}

Month II: Ayyaru

ii 1 SiA DIš ina ritiayyaru(GUD) UD.1.KAM úl magir (ŠE.GA) ${ }^{1}$

ii 2 SiA '2' magār(ŠE.GA) kabti(IDIM)

ii $3 \quad \mathrm{SiA}{ }^{1} 3^{1}{ }^{\prime} \bar{s}^{\prime} a$ (ŠE $)$ là inaddin(SUM.MU)

ii $4 \quad$ SiA illegible traces

ii $5 \quad$ SiA illegible trace

ii 6-7 $\quad \mathrm{SiA} \quad[\ldots]$

ii $8 \quad$ SiA 18 kabtu(IDIM) magir(

ii $9 \quad \mathrm{SiA} \quad$ i9 nūna $\left(\mathrm{KU}_{6}\right)$ là $i k k a l\left(\mathrm{GU}_{7}\right) \operatorname{murșu(GIG)}$ isabbat (DAB)-su?

ii $10 \quad \mathrm{SiA} \quad 10 \operatorname{dinu}(\mathrm{DI})$ magir (ŠE.GA)

ii $11 \quad \mathrm{SiA} 11 \operatorname{dinu(DI)~úl~magir(\breve {EE}.GA)}$

ii $12 \quad$ SiA 12 in sūqi(SILA) $\operatorname{magir}(\breve{\text { S[E.G]A) }}$

ii $13 \quad \mathrm{SiA} 13 \mathrm{ka}[s p a$ (KÙ.BABBAR) là $i p-p] e ́$-eš

SiB 13 K[Ù.BABBAR ... . ]

ii 14 SiA [14 pi-is]-la-tú

SiB $14 p i-[\ldots]$

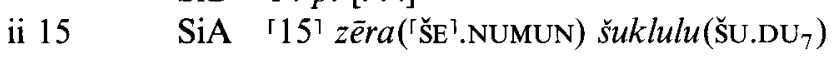

SiB 15 ŠE.N[UMUN ....]

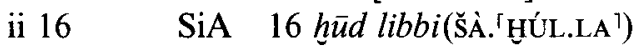

SiB 16 Š[À.HUUL.L]A

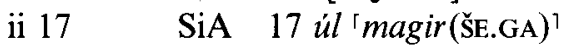

SiB 17 N[U.(UN)].ŠE.GA

ii $18 \quad \mathrm{SiA} 18 z[a$ ? $\ldots-$-šu $] r$ ?

$\mathrm{SiB} 18 z a-k u-t u ́[p] u-s ̌ u r$

ii $19 \quad$ SiA $19^{r} m a^{\top}-m i t ~ l a ̀ ~ ' i$ ? -tam?-ma?

SiB 19 ma-mi-tum là itamma(KUD)

ii 20 SiA $20{ }^{\top}$ MUŠ H̦É'.[. . .]

SiB $20 \operatorname{se} r a$ (MUŠ) lidūk (HÉ.EN.GAZ) ašarēeūta(SAG.KAL) illak(DU)

ii 23

ii 24

ii 25

ii 26

ii 27

ii 28

ii 29

ii 30
[18: wrongdoing] will depart

[19:] weeping
I In Ayyaru, day one: it is not favourable

2: favour of an important person

3: one must not barter grain

8: an important person is favourable

9: one must not eat fish: sickness might seize one

10: a verdict is favourable

11: a verdict is unfavourable

12: it is favourable in the street

13: [one must not] deal in silver

14: accidents

15: finished seed-corn(?)

16: happiness

17: it is unfavourable

18: undo exemption(?)

19: one must not swear an oath

20: one should kill a snake: one might become a leader

21: treachery

22: it is favourable in a lawsuit

23: discontent

24: happiness

25: one must not acquire a wife

[26:] news

[27:] slander

[28:] it is [completely] favourable

[29:] one should kill [a snake]

[30:] favourable [portent] 
Month III: Simānu

\begin{tabular}{|c|c|c|}
\hline iii 1 & $\mathrm{SiA}$ & 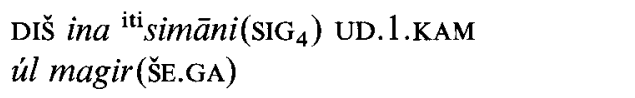 \\
\hline iii 2 & SiA & $2 \bar{u} m u(\mathrm{UD})$ là magru(ŠE.GA) \\
\hline iii 3 & $\mathrm{SiA}$ & $\begin{array}{l}3 \text { [še'a(ŠE)] pu-šsu-ur ilu(DINGIR) ana amèeli } \\
\text { (LÚ.RA) lumun(HUL)-šu ippattar(DUH.A) }\end{array}$ \\
\hline $\begin{array}{l}\text { iii } 4 \\
\text { iii } 5-10\end{array}$ & $\mathrm{SiA}$ & $\begin{array}{l}{\left[\begin{array}{ll}4 & \dot{u} l\end{array}\right] \text { magir }(\breve{\mathrm{SE}} . \mathrm{GA})} \\
\text { not extant }\end{array}$ \\
\hline 11 & $\mathrm{SiA}$ & $11[\ldots]$ \\
\hline 12 & $\mathrm{SiA}$ & 12 il sūqi(SILA) ina sūqi(SILA) [magir] \\
\hline \multirow[t]{2}{*}{ iii 13} & $\mathrm{SiA}$ & 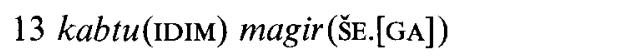 \\
\hline & $\mathrm{SiB}$ & $13[\ldots] \mathrm{x}$ \\
\hline \multirow[t]{2}{*}{ iii 14} & SiA & $14 \dot{u} l$ magir (šS.GA) \\
\hline & $\mathrm{SiB}$ & $14[\ldots] \mathrm{x}$ \\
\hline \multirow[t]{2}{*}{ iii 15} & $\mathrm{SiA}$ & 15 damassa(LAMMA) irašši(TUK) \\
\hline & $\mathrm{SiB}$ & $15^{\mathrm{d}} \mathrm{LAM}[\mathrm{MA}] \mathrm{TUK}$ \\
\hline \multirow[t]{2}{*}{ iii 16} & $\mathrm{SiA}$ & 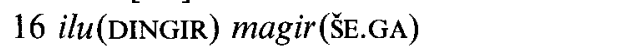 \\
\hline & $\mathrm{SiB}$ & 16 DINGIR ŠE.GA \\
\hline \multirow[t]{2}{*}{ iii 17} & SiA & 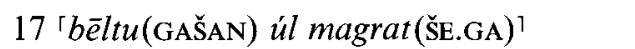 \\
\hline & $\mathrm{SiB}$ & 17 GAŠAN $u ́ l$ ŠE.GA \\
\hline \multirow[t]{2}{*}{ iii 18} & $\mathrm{SiA}$ & $18^{r} k a-a r^{1}-s i$ \\
\hline & $\mathrm{SiB}$ & $18 k a-a r-s i$ \\
\hline \multirow[t]{2}{*}{ iii 19} & SiA & $19^{\top} \dot{u} l$ magir $(\mathrm{ŠE} . \mathrm{GA})^{\top}$ \\
\hline & $\mathrm{SiB}$ & $19 u ́ l$ ŠE.GA \\
\hline \multirow[t]{2}{*}{ iii 20} & SiA & illegible traces \\
\hline & $\mathrm{SiB}$ & [20 antalê(AN.T]A.LÙ) d šamaš(UTU) \\
\hline iii 21 & $\mathrm{SiB}$ & [21 giš ]eleppa(MÁ) là irakkab $\left(\mathrm{U}_{5}\right)$ \\
\hline 22 & $\mathrm{SiB}$ & $2[2] z i$-it-tum \\
\hline 23 & $\mathrm{SiB}$ & 23 úl magir (ŠE.GA) \\
\hline 24 & $\mathrm{SiB}$ & 24 bēltu(GAŠAN) magrat(̌̌s.[GA]) \\
\hline 25 & $\mathrm{SiB}$ & 25 ilu(DINGIR) úl magir(ŠE.GA) \\
\hline 26 & $\mathrm{SiB}$ & 26 bëltu(GAŠAN) magrat(吕.GA) \\
\hline 27 & $\mathrm{SiB}$ & 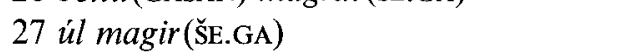 \\
\hline 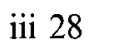 & $\mathrm{SiB}$ & 28 in $\operatorname{dini}(\mathrm{DI})$ magir(ŠE.GA) \\
\hline & $\mathrm{SiB}$ & 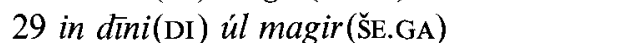 \\
\hline & $\mathrm{SiB}$ & 30 pi-is-la-tum \\
\hline
\end{tabular}

II In Simānu, day one: it is not favourable

2: unfavourable day

3: sell [grain]; a god's ill will toward a man will be undone [4: it is not] favourable

12: the god of the street [will be favourable] in the street

13: an important person will be favourable

14: it is unfavourable

15: one will acquire a guardian angel

16: a god is favourable

17: a mistress is unfavourable

18: slander

19: it is not favourable

[20: eclipse] of the sun

[21:] one should not go by boat

22: division of shares

23: it is unfavourable

24: a mistress is favourable

25: a god is unfavourable

26: a mistress is favourable

27: it is unfavourable

28: it is favourable in a lawsuit

29: it is unfavourable in a lawsuit

30: accidents

I In Dumuzi, day one: wrath of Šamaš

2: weeping

3: release a caged bird: one's evil will be undone from one 4: either attack by lion or attack by snake

[5: expenditure of] grain

iv $5 \quad \mathrm{SiA} \quad[5$ si-it s] $] e-e$

iv $6 \quad \mathrm{SiA} \quad[6 \ldots] \times \mathrm{x}$

iv 7-8 not extant (distributed over three lines on SiA)

iv $9 \quad$ SiA $[9 \ldots] \mathrm{x}$

iv $10 \quad$ SiA $\quad\left[\begin{array}{lll}10 & \text { ana }\end{array}{ }^{\lceil} \text {sūqi (SILA) }\right)^{\top}$ là ussi( (I.B]Í.ZA immar(IGI)

iv $11 \quad \mathrm{SiA} 11 \mathrm{ka}$-liš magir(

[10:] one must not go out [on to] the street: one might experience loss 11: it is completely favourable 


\begin{tabular}{|c|c|c|}
\hline \multirow[t]{2}{*}{ iv 12} & $\mathrm{SiA}$ & 12 ša-se-e šarri(LUGAL) \\
\hline & $\mathrm{SiB}$ & $12 \times[\ldots]$ \\
\hline \multirow[t]{2}{*}{ iv 13} & $\mathrm{SiA}$ & 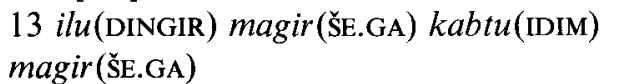 \\
\hline & $\mathrm{SiB}$ & 13. \\
\hline \multirow[t]{2}{*}{ iv 14} & $\mathrm{SiA}$ & 14 ilu(DINGIR) úl magir(ŠE.GA) \\
\hline & $\mathrm{SiB}$ & 14. \\
\hline \multirow[t]{2}{*}{ iv 15} & $\mathrm{SiA}$ & 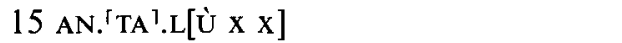 \\
\hline & $\mathrm{SiB}$ & 15 attalê(AN.TA.LÙ) ${ }^{\mathrm{d}} \sin (30)$ \\
\hline \multirow[t]{2}{*}{ iv 16} & $\mathrm{SiA}$ & $16 \times[\ldots]$ \\
\hline & $\mathrm{SiB}$ & 16 ni-pi-ih išāti(IZI) \\
\hline \multirow[t]{2}{*}{ iv 17} & $\mathrm{SiA}$ & $17[\ldots]$ \\
\hline & $\mathrm{SiB}$ & $\begin{array}{l}17 \text { dammasu(LAMMA) [magrat magār dīni } \\
\text { zakār šu]-mi }\end{array}$ \\
\hline iv 18 & $\mathrm{SiB}$ & 18 gi-li-it-tum harrāni(KASKAL) \\
\hline iv 19 & $\mathrm{SiB}$ & 19 magār(ŠE.GA) šarri(LUGAL.LA.KAM) \\
\hline iv 20 & $\mathrm{SiB}$ & $20 \bar{u} m u(\mathrm{UD})$ là magru(ŠE.GA) \\
\hline iv 21 & $\mathrm{SiB}$ & 21 magār(ŠSE.GA) šarri(LUGAL.LA.KAM) \\
\hline iv 22 & $\mathrm{SiB}$ & 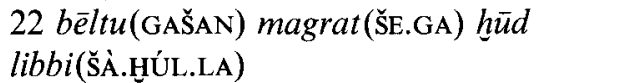 \\
\hline iv 23 & $\mathrm{SiB}$ & 23 gaba-ra-ah \\
\hline iv 24 & $\mathrm{SiB}$ & $24 z e-n u-u t$ ah-he-e \\
\hline iv 25 & $\mathrm{SiB}$ & 25 amēlu(LÚ) la i-tam-ma \\
\hline iv 26 & $\mathrm{SiB}$ & 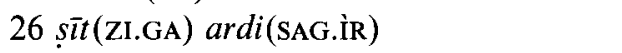 \\
\hline iv 27 & $\mathrm{SiB}$ & 27 in dini(DI) ma-gir \\
\hline iv 28 & $\mathrm{SiB}$ & 28 ka-liš ma-gir \\
\hline iv 29 & $\mathrm{SiB}$ & 29 ka-liš ma-gir \\
\hline iv 30 & $\mathrm{SiB}$ & 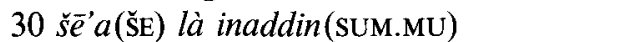 \\
\hline
\end{tabular}

Month V: Abu

\begin{tabular}{|c|c|c|}
\hline v 1 & $\mathrm{SiA}$ & $\begin{array}{l}\text { DIŠ ina }{ }^{\text {iti }} a b u(\mathrm{NE}) \text { UD.1.KAM } b \bar{e} l t u(\mathrm{GAŠAN}) \\
\text { magrat( } \text { (ŠE.GA) }\end{array}$ \\
\hline v 2 & $\mathrm{SiA}$ & 2 NÍG.BA $i l i$ (DINGIR) \\
\hline v 3 & $\mathrm{SiA}$ & $3 b i-k i-t u m$ \\
\hline v 4 & $\mathrm{SiA}$ & 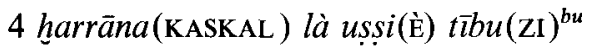 \\
\hline v 5 & SiA & 5 mišil(BAR) ümi(UD) magir(吕.GA) \\
\hline v 6 & $\mathrm{SiA}$ & $6 t \bar{l} b u(\mathrm{ZI})^{b u}$ \\
\hline v 7 & $\mathrm{SiA}$ & 7 ūmu(UD) là magru(ŠE.GA) \\
\hline v 8 & SiA & ${ }^{1} 8^{7} \times \times \times \times x \times$ \\
\hline v 9 & $\mathrm{SiA}$ & $\begin{array}{l}\text { [9] 'kurummat (ك̌UK)-su?' ana ' } g u-l a \\
\text { liškun(GAR) }\end{array}$ \\
\hline v 10 & $\mathrm{SiA}$ & $10 \bar{u} m u(\mathrm{UD}) \operatorname{magru}(\mathrm{S} \mathrm{E} . \mathrm{GA})$ \\
\hline v 11 & $\mathrm{SiA}$ & $11 \bar{u} m u(\mathrm{UD})$ là magru(ŠE.GA) \\
\hline v 12 & SiA & $12 \bar{u} m u(\mathrm{UD})$ là magru(ŠE.GA) \\
\hline v 13 & $\mathrm{SiA}$ & $13 \bar{u} m u(\mathrm{UD})$ là magru(ŠE.GA) \\
\hline \multirow[t]{2}{*}{ v 14} & $\mathrm{SiA}$ & $14 \bar{u} m u(\mathrm{UD})$ là magru(ŠSE.GA) \\
\hline & $\mathrm{SiB}$ & $14 \mathrm{U}[\mathrm{D} \ldots]$ \\
\hline \multirow[t]{2}{*}{ v 15} & $\mathrm{SiA}$ & $15 \bar{u} m u($ UD $)$ magru(šE.GA) \\
\hline & $\mathrm{SiB}$ & 15 UD $[\ldots]$ \\
\hline \multirow[t]{2}{*}{ v 16} & SiA & $16 \bar{u} m u(\mathrm{UD})$ magru(ŠE.GA) \\
\hline & $\mathrm{SiB}$ & 16 UD $[\ldots]$ \\
\hline \multirow[t]{2}{*}{ v 17} & $\mathrm{SiA}$ & 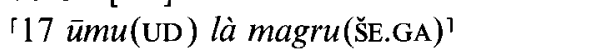 \\
\hline & $\mathrm{SiB}$ & $17[\ldots]$ \\
\hline
\end{tabular}

12: summons by the king

13: a god is favourable, an important person is favourable

14: a god is unfavourable

15: eclipse of the moon

16: outbreak of fire

17: a guardian spirit [is favourable; favour at law, calling by] name

18: fright on the road

19: favour by the king

20: unfavourable day

21: favour by the king

22: a mistress is favourable:

happiness

23: revolt

24: feud among brothers

25: a man must not take an oath

26: escape of a slave

27: it is favourable in a lawsuit

28: it is completely favourable

29: it is completely favourable

30: one must not barter grain

If In Abu, day one: a mistress is favourable

2: gift to a god (or wrath of god)

3: weeping

4: one must not set out on a journey: attack

5: midday is favourable

6: attack

7: unfavourable day

[9:] one should present one's food to Gula

10: favourable day

11: unfavourable day

12: unfavourable day

13: unfavourable day

14: unfavourable day

15: favourable day

16: favourable day

17: unfavourable day 


\begin{tabular}{|c|c|c|}
\hline v 18 & $\mathrm{SiB}$ & $18[\ldots]$ \\
\hline v 19 & $\mathrm{SiB}$ & $19[\ldots]$ \\
\hline v 20 & $\mathrm{SiB}$ & $20[\ldots]$ \\
\hline v 21 & $\mathrm{SiB}$ & $2[1 \quad \bar{u} m u(\mathrm{UD})$ là magru(ŠE.G]A) \\
\hline v 22 & $\mathrm{SiB}$ & $2[2 \bar{u} m u(\mathrm{UD}) l] \grave{a}$ magru(ŠE.GA) \\
\hline v 23 & $\mathrm{SiB}$ & [23 ümu(UD) l]à magru(ŠE.GA) \\
\hline v 24 & $\mathrm{SiB}$ & 24 [hil]-bil-tum \\
\hline v 25 & $\mathrm{SiB}$ & 25 gaba-ra-ah \\
\hline v 26 & $\mathrm{SiB}$ & $26 \bar{u} m u(\mathrm{UD})[l] \grave{a} \operatorname{magru}(\breve{\mathrm{SE}} . \mathrm{GA})$ \\
\hline v 27 & $\mathrm{SiB}$ & 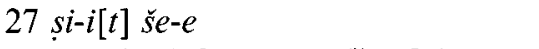 \\
\hline v 28 & $\mathrm{SiB}$ & $28 \ddot{u} m u(\mathrm{UD}) l[\grave{a} \operatorname{magru}($ ŠE.G $] \mathrm{A})$ \\
\hline v 29 & $\mathrm{SiB}$ & $29 \bar{u} m u$ (UD) $l[a ̀ \operatorname{magru(Š\mathrm {E}.\mathrm {G}]\mathrm {A})}$ \\
\hline v 30 & $\mathrm{SiB}$ & 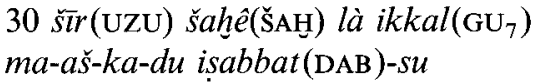 \\
\hline
\end{tabular}

Month VI: Ulūlu

\begin{tabular}{|c|c|c|}
\hline vi 1 & $\mathrm{SiA}$ & DIš ina ${ }^{\text {iti }} u l u \bar{l} l i(\mathrm{KIN})$ UD.1.KAM x $[\mathrm{x}]$ \\
\hline vi 2 & $\mathrm{SiA}$ & 2 magir (AB.ŠE.GE.D $[\mathrm{A}])$ \\
\hline vi 3 & SiA & 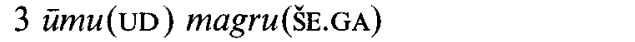 \\
\hline vi 4 & $\mathrm{SiA}$ & $4 \bar{u} m u(\mathrm{UD})$ là magru(ŠSE.GA) \\
\hline vi 5 & SiA & 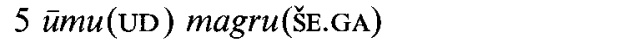 \\
\hline vi 6 & $\mathrm{SiA}$ & $6 \bar{u} m u(\mathrm{UD})$ là magru(ŠE.G[A]) \\
\hline vi 7 & $\mathrm{SiA}$ & $7 \bar{u} m u(\mathrm{UD}) \operatorname{magru(Š\mathrm {E}.GA)}$ \\
\hline vi 8 & SiA & '81 șalta(LÚ.NE) lā ippušs?(NA.[AN.DÙ.DÙ?]) \\
\hline vi 9 & $\mathrm{SiA}$ & 9 șabāt eqli(A.š̀̀ AN.[DAB.BA]) \\
\hline vi 10 & $\mathrm{SiA}$ & $10 \bar{u} m u(\mathrm{UD}) l \grave{a}[\mathrm{magru}(\mathrm{S} \mathrm{E} . \mathrm{GA})]$ \\
\hline vi 11 & $\mathrm{SiA}$ & $11 \bar{u} m u(\mathrm{UD}) \operatorname{magru(\breve {\mathrm {S}}.[\mathrm {GA}])}$ \\
\hline vi 12 & SiA & $12 \bar{u} m u(\mathrm{UD})$ là magru( $\mathrm{\textrm {SE }} \cdot[\mathrm{GA}])$ \\
\hline vi 13 & $\mathrm{SiA}$ & $13 n i-z i[q-t u m]$ \\
\hline vi 14 & $\mathrm{SiA}$ & $14 h u-u[d l i b-b i]$ \\
\hline vi 15 & $\mathrm{SiA}$ & $15^{\ulcorner} \bar{u} m u(\mathrm{UD})^{\urcorner}\left[\operatorname{magru}\left(\check{S}^{\mathrm{S}} . \mathrm{GA}\right)\right]$ \\
\hline vi 16 & & not extant on either source \\
\hline vi 17 & $\mathrm{SiB}$ & {$[17 \mathrm{x}] \times[\ldots]$} \\
\hline vi 18 & $\mathrm{SiB}$ & 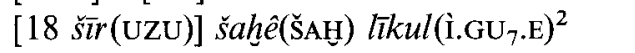 \\
\hline vi 19 & $\mathrm{SiB}$ & {$[19 b u]-s u ́ u-r a-t u m$} \\
\hline vi 20 & $\mathrm{SiB}$ & [20 sa]-ba-at ${ }^{\text {giš }} k a k k i$ (TUKUL) \\
\hline vi 21 & $\mathrm{SiB}$ & $21 \dot{\bar{u}} m u(\mathrm{UD}) \operatorname{magru}($ ŠE.GA) \\
\hline vi 22 & $\mathrm{SiB}$ & 22 ümu(UD) là magru(šs.GA) \\
\hline vi 23 & $\mathrm{SiB}$ & $23 \bar{u} m u(\mathrm{UD})$ magru(ŠE.GA) \\
\hline vi 24 & $\mathrm{SiB}$ & 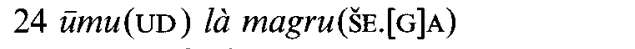 \\
\hline vi 25 & $\mathrm{SiB}$ & 25 amēlu(LÚ) là i-tam-má \\
\hline vi 26 & $\mathrm{SiB}$ & 26 mi-im-ma la i-šá-am \\
\hline vi 27 & $\mathrm{SiB}$ & 27 ūmu(UD) là magru(ŠE.GA) \\
\hline vi 28 & $\mathrm{SiB}$ & 28 ri-gim ki-di-im \\
\hline 29 & $\mathrm{SiB}$ & $\begin{array}{l}29 k a-s i-a \text { pu-tur } \\
\text { ernetta(Ù.MA) kušud(SÁ.SÁ) }\end{array}$ \\
\hline & $\mathrm{SiB}$ & 30 ki-mil-ti ili(DINGIR) \\
\hline
\end{tabular}

Month VII: Tašrītu

\begin{tabular}{|c|c|c|}
\hline vii 1 & $\mathrm{SiB}$ & $\begin{array}{l}\text { DIŠ ina }{ }^{\text {iti }} \text { tašrñti } i\left(\mathrm{DU}_{6} . \mathrm{KU}\right) \\
\text { UD.1.KAM UD ŠE.GA }\end{array}$ \\
\hline vii 2 & $\mathrm{SiB}$ & 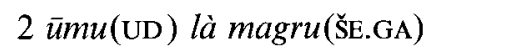 \\
\hline vii 3 & $\mathrm{SiB}$ & $3 n \bar{u} n a\left(\mathrm{KU}_{6}\right) l \bar{a} i k k a l\left(\mathrm{NA} . \mathrm{AN} . \mathrm{GU}_{7} . \mathrm{E}\right)$ \\
\hline
\end{tabular}

\author{
21: [unfavourable day] \\ 22: unfavourable [day] \\ [23:] unfavourable [day] \\ 24: wrongdoing \\ 25: revolt \\ 26: unfavourable day \\ 27: loss of grain \\ 28: unfavourable day \\ 29: unfavourable day \\ 30: one must not eat pig meat: \\ maškadu-sickness might seize one
}

T In Ulūlu, day one: [ . . ]

2: it is favourable

3: favourable day

4: unfavourable day

5: favourable day

6: unfavourable day

7: favourable day

8: one must not quarrel

9: seizure of a field

10: unfavourable day

11: favourable day

12: unfavourable day

13: anxiety

14: happiness

15: [favourable] day

[18:] one should eat pig [meat]

[19:] news

[20:] taking up weapons

21: favourable day

22: unfavourable day

23: favourable day

24: unfavourable day

25: a man must not take an oath

26: one must not buy anything

27: unfavourable day

28: uproar outside

29: release a captive, achieve an aim

30: wrath of a god

TI In Tašritu, day one:

favourable day

2: unfavourable day

3: one must not eat fish 
vii 4

vii 5

vii 6

vii 7

vii 8

vii 9

vii 10

vii 11

vii 12

vii 13

vii 14

vii 15

vii $16-17$

vii 18

vii 19

vii 20

vii 21

vii 22

vii 23

vii 24

vii 25

vii 26

vii 27

vii 28

vii 29

vii 30
$\mathrm{SiB} \quad 4[$ ișsūura(MUŠEN) șabta(DAB) l]imǎ̌šer (HÉ.EN.BAR) ki-mil-tim ippattar(DUH)

$\mathrm{SiB} 5$ karaša $\left(\mathrm{GA}[\mathrm{RAŠs})^{\mathrm{s}}\right]^{\mathrm{ar}} l \bar{a}$ ikkal(NA.AN.GU $\left.7 . \mathrm{E}\right)$

SiB $6[\bar{u} m u$ (UD) (là)] magru( (̌̆E.GA)

$\mathrm{SiB} 7$ in dini(DI) [ú]l magir(

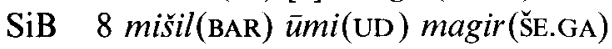

SiB 9 in dīni(DI) úl magir (ŠE.GA)

$\mathrm{SiB} 10 \bar{u} m u$ (UD) là magru(ŠE.GA)

$\mathrm{SiB} 11 \bar{u} m u$ (UD) magru(ŠE.GA)

SiB $12 \bar{u} m u$ (UD) là magru(ŠS.GA)

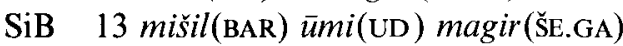

$\mathrm{SiB} 14 \bar{u} m u$ (UD) magru(ŠE.GA)

$\mathrm{SiB} 15$ aššata(DAM) [lā irašši $]$ not extant on either source

SiA $18 \bar{u} m u$ (UD) [là magru(šE.GA)]

SiA $19 i$-zi-[im-tú ikaššad(KUR)]

$\mathrm{SiA} 20$ attalê( $\left.\mathrm{AN} . \mathrm{GI}_{6}\right){ }^{\mathrm{d}}[\mathrm{x}]$

SiA 21 ma-gàr [šs $’ i(\breve{\mathrm{S}})]$

SiA 22 magār (ك̌E.GA) šarri(LUGAL.LA?)

SiA 23 magār (ŠE.GA) [šarri]

SiA $24 \bar{u} m u$ (UD) là magru(šE.GA)

SiA 25 ša-se!-e [šarri]

SiA 26 kabtu(IDIM) magir( (̌̌.GA)

Month VIII: Arahsamnu

viii 1

$\mathrm{SiB}$ DIš ina ${ }^{\text {iti }}$ arahsamni(APIN.DUH.A)

UD.1.KAM úl magir( $\breve{E} . \mathrm{GA})$

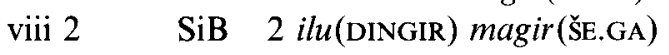

viii $3 \quad \mathrm{SiB} \quad 3 s_{s} \bar{e}^{\prime} a$ (ŠE) là inaddin (SUM.MU)

viii $4 \quad \mathrm{SiB} \quad 4 s \bar{u} q$ (SILA) $i b \bar{a} u(\mathrm{DIB}) l a i-b a$-' $^{\prime}$

viii $5 \quad \mathrm{SiB} \quad 5$ amēlu(LÚ) aššata(DAM) là irašši(TUK)

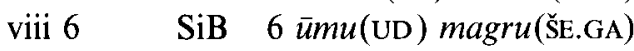

viii $7 \quad \mathrm{SiB} \quad 7 \bar{u} m u$ (UD) magru(S̆E.GA)

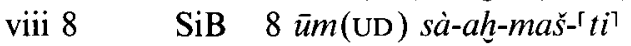

viii $9 \quad \mathrm{SiB} \quad 9 \bar{u} m u(\mathrm{UD}) \operatorname{magru}(\mathrm{S}[\mathrm{E} . \mathrm{GA}])$

viii $10 \quad \mathrm{SiB} 10$ suluppī(zú.Lum) lā

$i k k a l\left(\right.$ NU.UN. $\left.\left[\mathrm{GU}_{7} .(\mathrm{E})\right]\right)$

viii $11 \quad \mathrm{SiB} \quad 11 \bar{u} m u(\mathrm{UD}) \operatorname{magru}\left(\mathrm{SE}_{\mathrm{E}}[\mathrm{GA}]\right)$

viii $12 \quad \mathrm{SiB} 12 \mathrm{LAGAB} \times \mathrm{A}^{3} \mathrm{x}^{3}$ là inaddin(SUM.MU) úl iššir (SI)?-šu?

viii $13 \quad \mathrm{SiB} 13 \bar{u} m u(\mathrm{UD}) \operatorname{magru}(\check{\mathrm{S} E} . \mathrm{GA})$

viii $14 \quad \mathrm{SiB} 14 \bar{u} m u(\mathrm{UD})$ là magru(šE.GA)

viii $15 \quad \mathrm{SiB} 15 \bar{u} m u(\mathrm{UD}) \operatorname{magru}(\mathrm{S} \mathrm{E} . \mathrm{GA})$

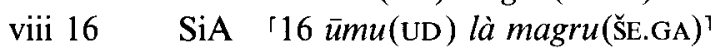

SiB 16 UD ŠE.GA
4: one should free [a caged bird,]

anger will be assuaged

5: one must not eat leeks

6: [(un)] favourable [day]

7: it is unfavourable in court

8: midday is favourable

9: it is unfavourable in court

10: unfavourable day

11: favourable day

12: unfavourable day

13: midday is favourable

14: favourable day

15: [one must not acquire] a wife

18: [unfavourable] day

19: [one will obtain a] desire

20: eclipse of the [sun/moon]

21: favour of [grain]

22: favour by the king

23: favour by the king

24: unfavourable day

25: summons by [the king]

26: an important person is

favourable

27: one must not eat ox meat

[...]

28: achieve an aim

29: it is completely favourable

30: accidents

II In Arahsamnu, day one:

it is unfavourable

2: a god is favourable

3: one must not barter grain

4: one must not go down a street

that one went down (before)

5: a man must not acquire a wife

6: favourable day

7: favourable day

8: day of uprising

9: favourable day

10: one must not eat dates

11: favourable day

12: one must not barter...,

it will not bring one profit

13: favourable day

14: unfavourable day

15: favourable day

16: unfavourable day ( $\mathrm{SiA})$

favourable day $(\mathrm{SiB})$

\footnotetext{
${ }^{3}$ We expect kaspa(KÙ.BABBAR), but ef. ix 3 .
} 


\author{
viii $17 \quad$ SiA $17 \bar{u} m$ (UD) șal-tum \\ SiB 17 UD sa-al-tum? \\ viii $18 \quad \mathrm{SiA} \quad 18 \bar{u} m u$ (UD) $\operatorname{magru}$ (吕.GA) \\ SiB 18 UD [...] \\ viii $19 \quad \mathrm{SiA} 19$ ki-mil-ti murși(GIG) ippațtar(DUH)

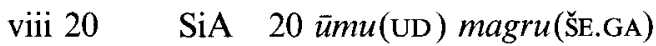 \\ viii $21 \quad$ SiA $21 \bar{u} m u$ (UD) là magru(ŠE.GA) \\ viii 22 SiA 22 hu-ud libbi(š̀) \\ viii 23 SiA [23] ūmu(UD) là magru(ŠE.GA) \\ viii 24 \\ viii 25 \\ viii 26 \\ viii 27 \\ SiA $\left\ulcorner 24\right.$ bu-sú-ra-tum ${ }^{\prime}$ \\ SiA ${ }^{2} 25 \bar{u} m u(\mathrm{UD})$ là magru(ŠE.GA) ${ }^{1}$ \\ SiA $26 i l u$ (DINGIR) magir( $($ SE.GA) \\ SiA $27 s \bar{u} q$ (SILA) $i b \bar{a}^{\prime} u$ (DIB) là $i b \hat{a}^{\prime}$ (DIB) \\ $t \bar{\imath} b u(\mathrm{ZI})^{b u}$
}

viii 28

viii 29

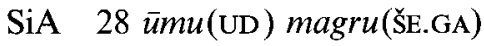

SiA 29 ana $\sin (30)$ là $u s ̌-k e-e n$ kurummat(ธ̌UK)-su ana $\sin (30)$ išakkan (GAR)

viii $30 \quad \mathrm{SiA} \quad 30 \mathrm{ka}$-liš magir(ŠE.GA)

Month IX: Kislīmu

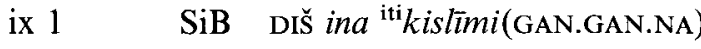

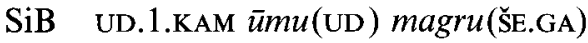

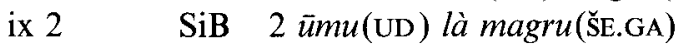

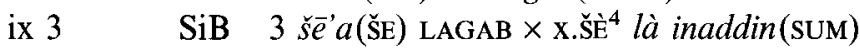
$\mathrm{x} \times \mathrm{x} \times$ - $t$ im

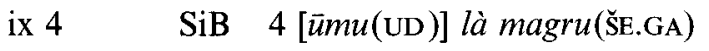

ix 5-7 $\quad \mathrm{SiB}$ illegible traces

ix $8 \quad \mathrm{SiB} \quad 8[\bar{u} m u(\mathrm{UD})]$ là magru(ŠE.GA)

ix $9 \quad \mathrm{SiB} \quad 9 \bar{u} m u(\mathrm{UD}) \operatorname{magru}(\check{\mathrm{S}} \mathrm{E} . \mathrm{GA})$

ix $10 \quad \mathrm{SiB} \quad 10 \bar{u} m u$ (UD) là magru( (SE.GA)

ix $11 \quad \mathrm{SiB} 11 \bar{u} m u$ (UD) magru(

ix 12 SiB $12 \times \times \times \times \times \times \operatorname{immar}($ IGI)

ix $13 \quad \mathrm{SiA} 13^{\mathrm{I}} \mathrm{UD}^{1}[\ldots]$

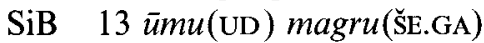

ix $14 \quad \mathrm{SiA} 14$ UD [...]

SiB 14 ūmu(UD) là magru( (̌̌E.GA)

ix 15 SiA 15 SILA DIB [...]

SiB 15 sūq(SILA) ibā'u(DIB) là ibâ'(DIB)

ix $16 \quad \mathrm{SiA} 16 \bar{u} m u$ (UD) magru(ŠE.GA)

SiB 16 UD ŠE.GA

ix $17 \quad$ SiA $17 \bar{u} m u$ (UD) là magru(ŠE.GA)

SiB 17 UD là ŠE.GA

ix $18 \quad$ SiA 18 ka-liš magir(ŠE.GA)

$\mathrm{SiB} 18$ UD [(x)] ŠE.GA

ix 19 SiA $\left\ulcorner 19 t a^{\top}\right.$-nu-qat șâlti(LÚ.NE?) ibašši(GÁL)

$\mathrm{SiB} 19 \times \mathrm{x}[\ldots] \mathrm{x} \times$

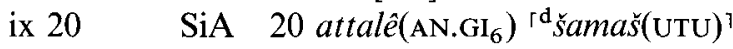

$\mathrm{SiB} 20$ AN.T[A.LÙ ....]

ix $21 \quad$ SiA $21 \bar{u} m u$ (UD) magru(ŠE.GA)
17: day of affray

18: favourable day

19: anger (that causes) sickness

will be assuaged

20: favourable day

21: unfavourable day

22: happiness

[23:] unfavourable day

24: news

25: unfavourable day

26: a god is favourable

27: one must not go down a street

that one went down (before):

attack

28: favourable day

29: one must not bow down to

Sîn; one must present one's food to him

30 : it is completely favourable

I In Kislìmu, day one: favourable day

2: unfavourable day

3: one must not barter grain for

...

4: unfavourable [day]

8: unfavourable [day]

9: favourable day

10: unfavourable day

11: favourable day

12: one will see...

13: favourable day

14: unfavourable day

15: one must not go down a street that one went down (before)

16: favourable day

17: unfavourable day

18: it is completely favourable

19: hue and cry of affray will

occur

20: eclipse of the sun

21: favourable day 


\begin{tabular}{|c|c|c|}
\hline ix 22 & $\mathrm{SiA}$ & {$[2] 2^{\ulcorner} \bar{u} m u(\mathrm{UD}) l_{a}{ }^{1}$ magru(ŠE.GA) } \\
\hline ix 23 & $\mathrm{SiA}$ & {$[23 \bar{u} m u(\mathrm{UD})]^{\lceil} \operatorname{magru}\left(\check{S}_{\mathrm{E} E G \mathrm{GA}}{ }^{\top}\right.$} \\
\hline ix 24 & $\mathrm{SiA}$ & 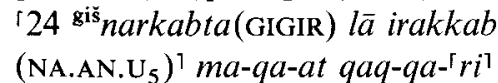 \\
\hline ix 2 & $\mathrm{SiA}$ & $25 \bar{u} m u($ UD $)$ magru(ŠE.GA) \\
\hline ix 2 & $\mathrm{SiA}$ & $26 \bar{u} m u(\mathrm{UD})$ là magru(ŠE.GA) \\
\hline ix 2 & $\mathrm{SiA}$ & 27 ni-is-sà-tum \\
\hline & $\mathrm{SiA}$ & 28 aššata(DAM) là irašši(TUK) \\
\hline & $\mathrm{SiA}$ & 29 ka-liš magir(ŠE.GA) \\
\hline & $\mathrm{SiA}$ & $30 \bar{u} m u(\mathrm{UD})$ magru(ŠSE.GA) \\
\hline
\end{tabular}

Month X: Tebētu

$\mathrm{x} 1-1$

$\mathrm{x} 12$

$\mathrm{x} 13$

x 14

x 15

$\mathrm{x} 16$

x 17

x 18

x 19

x 20

$\mathrm{x} 21$

$\mathrm{x} 22$

$\mathrm{x} 23$

$\mathrm{x} 24$

$\times 25$

$\times 26$

x 27

x 28

$\times 29$

x 30 not extant on SiA

SiA $12 \times \times \times x$

SiA $13 \bar{u} m u$ (UD) magru(ŠE.GA)

SiA $14 \bar{u} m u(\mathrm{UD}) \operatorname{magru}($ ŠE.GA)

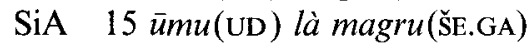

SiA $16 \breve{s} \bar{e} a(\breve{S} E)$ là inaddin(SUM) HA.LAM

SiA $17{ }^{\prime}$ mišil(BAR)' ümi(UD) magir(ŠE.GA)

SiA 18 ümu(UD) là magru(ŠE.GA)

SiA ' 19 sīt(ZI.GA) ardi(SAG.íR)

[kaspu(KÙ.BABBAR.RA.KE 4 ) iha]lliq?

([Ú.GÙ B]A.AN.'DÉ')

$\mathrm{SiA} \quad[20 \ldots]$

SiA $[21 \ldots]$

SiA $2[2 \ldots]$

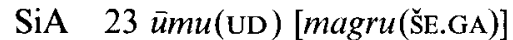

SiA 24 mišil(BAR) ūmi(UD) magir(ŠE.GA)

SiA 25 sa-li-mu

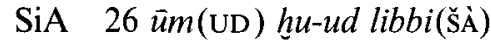

SiA 27 mišil(BAR) ūmi(UD) magir(ŠE.GA)

SiA $28 \bar{u} m u$ (UD) ${ }^{\text {he-p? }}$ magru(ŠE.GA)

SiA $29 \bar{u} m u$ (UD) magru( $\breve{E} . G A)$

SiA 30 ümu(UD) là magru(
22: unfavourable day

[23:] favourable [day]

24: one must not go by chariot:

fall to the ground

25: favourable day

26: unfavourable day

27: grief

28: one must not acquire a wife

29: it is completely favourable

30: favourable day

13: favourable day

14: favourable day

15: unfavourable day

16: one must not barter grain: ruin

17: midday is favourable

18: unfavourable day

19: escape of slave,

[silver will go] missing

23: [favourable] day

24: midday is favourable

25: friendship

26: day of happiness

27: midday is favourable

28: (un)favourable day

29: favourable day

30: unfavourable day

Month XI: Šabātu

\begin{tabular}{|c|c|c|}
\hline xi $1-11$ & & not extant on $\mathrm{SiA}$ \\
\hline xi 12 & $\mathrm{SiA}$ & $12 \bar{u} m u(\mathrm{UD})$ là magru(š[E.GA $])$ \\
\hline xi 13 & $\mathrm{SiA}$ & 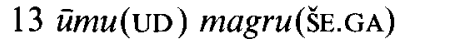 \\
\hline xi 14 & $\mathrm{SiA}$ & $14 \bar{u} m u$ (UD) magru(ŠE.GA) \\
\hline xi 15 & $\mathrm{SiA}$ & 15 ümu(UD) là magru(ŠE.GA) \\
\hline xi 16 & $\mathrm{SiA}$ & $16 \bar{u} m u(\mathrm{UD})$ là magru(ŠE.GA) \\
\hline xi 17 & $\mathrm{SiA}$ & ${ }^{\ulcorner} 17^{\prime} \bar{u} m u(\mathrm{UD})$ magru(ŠE.GA) \\
\hline xi 18 & $\mathrm{SiA}$ & {$[18 \bar{u} m u(\mathrm{UD}) l] \grave{a}$ magru(ŠSE.GA) } \\
\hline xi 19 & $\mathrm{SiA}$ & {$[19 \bar{u} m(\mathrm{UD}) h u]-u d \operatorname{libbi}(\widetilde{\mathrm{S}} \grave{\mathrm{A}})$} \\
\hline xi 20 & $\mathrm{SiA}$ & {$[20 b a-a] r-t u m$} \\
\hline xi 21 & $\mathrm{SiA}$ & 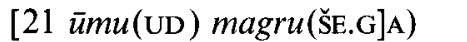 \\
\hline xi 22 & $\mathrm{SiA}$ & {$[22 \ldots]$} \\
\hline xi 23 & $\mathrm{SiA}$ & $2[3 \bar{u} m u(\mathrm{UD})] \operatorname{magru}(\check{\mathrm{SE}} .[\mathrm{GA}])$ \\
\hline xi 24 & $\mathrm{SiA}$ & 24 lib-bi lát tāabu(DÙG.GA) \\
\hline xi 25 & $\mathrm{SiA}$ & $25 \bar{u} m u$ (UD) magru(ŠE.GA) \\
\hline xi 2 & $\mathrm{SiA}$ & $26 \bar{u} m$ (UD) $h u-u d \operatorname{libbi(\mathrm {S}\check {\mathrm {A}})}$ \\
\hline xi 27 & $\mathrm{SiA}$ & $27 \bar{u} m u$ (UD) là magru(ŠE.GA) \\
\hline
\end{tabular}

12: unfavourable day

13: favourable day

14: favourable day

15: unfavourable day

16: unfavourable day

17: favourable day

[18:] unfavourable [day]

[19: day of ] happiness

[20:] rebellion

[21: favourable day]

23: favourable [day]

24: discontent

25: favourable day

26: day of happiness

27: unfavourable day

\footnotetext{
${ }^{5}$ Perhaps partially erased; we expect $l i b b u t a \bar{a} b u$, "contentment".
} 
xi $28 \quad$ SiA $28 \bar{u} m u(\mathrm{UD}) \operatorname{magru}(\breve{\mathrm{S} E} . \mathrm{GA})$

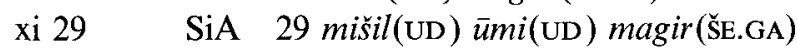

xi $30 \quad$ SiA 30 pi-is-la-tum

Month XII: Addāru

xii $1-11$ not extant on SiA

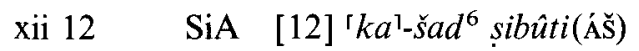

xii $13 \quad \mathrm{SiA}$ [1]3 ūmu(UD) là magru(ŠE.GA)

xii $14 \quad \mathrm{SiA} 14 \bar{u} m u$ (UD) là magru(ŠE.GA)

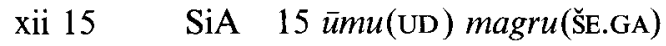

xii $16 \quad$ SiA $16 \bar{u} m u$ (UD) magru(ŠE.GA)

xii $17 \quad$ SiA $17 \bar{u} m u$ (UD) [là magru(ŠE.GA)]

xii 18 SiA 18 hi-bil-t[um]

xii $19 \quad$ SiA $19 \bar{u} m u(\mathrm{UD})$ là magru(ŠE.G[A])

xii $20 \quad$ SiA 20 magir( $\breve{s E .[G A])}$

xii 21 SiA $21 \bar{u} m u(\mathrm{UD})$ là magru(

xii $22 \quad$ SiA $22 \bar{u} m u(\mathrm{UD}) \operatorname{magru}(\breve{\mathrm{S}} \mathrm{E})$

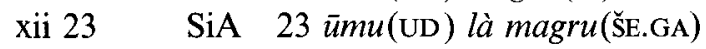

xii $24 \quad$ SiA 24 zitta(HA.LA) liqű̌s?(BA)

xii $25 \quad \mathrm{SiA} 25 \bar{u} m u$ (UD) magru( $(\breve{\mathrm{S}})$

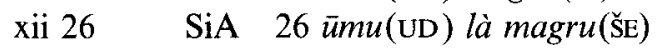

xii $27 \quad$ SiA $27 \bar{u} m u$ (UD) magru(ŠE)

xii $28 \quad \mathrm{SiA} 28 \bar{u} m u(\mathrm{UD})$ là magru( $\check{\mathrm{S} E})$

xii $29 \quad$ SiA $29 \bar{u} m u(\mathrm{UD})$ là magru( $\breve{\mathrm{S} E})$

xii $30 \quad$ SiA 30 ka-liš magir(ŠE.GA)

edge SiA unread colophon
28: favourable day

29: midday is favourable

30: accidents

[12:] success of enterprise

13: unfavourable day

14: unfavourable day

15: favourable day

16: favourable day

17: [favourable] day

18: wrongdoing

19: unfavourable day

20: it is favourable

21: unfavourable day

22: favourable day

23: unfavourable day

24: one should donate a share

25: favourable day

26: unfavourable day

27: favourable day

28: unfavourable day

29: unfavourable day

30 : it is completely favourable 